\title{
MALASSEZIA VERSUS CANDIDA IN HEALTHY DOGS
}

\author{
Sihelská, Z., Pangrácová Piterová, M., Čonková, E. \\ Harčárová, M., Böhmová, E. \\ Department of Pharmacology and Toxicology, \\ University of Veterinary Medicine and Pharmacy, Komenského 73, 04181 Košice \\ Slovakia \\ zuzana.sihelska@uvlf.sk
}

\section{ABSTRACT}

The genera Malassezia and Candida include yeasts which are members of the normal mycobiota of the skin and mucosal sites of humans and other warm-blooded animals. These yeasts are associated with a variety of dermatological disorders and also systemic diseases in humans and other animals. This study confirms the occurrence of Malassezia and Candida species in healthy dogs. Samples were collected from different body sites: external ear canal, interdigital area, skin of the axilla and of the neck, and the oral and rectal mucosae. The isolates were identified using phenotypic methods (biochemicalphysiological and morphological characteristics). The presence of yeasts were investigated in the specimens from 70 healthy dogs. Malassezia species were isolated in 44 dogs from which 84 Malassezia isolates were obtained. Only one Candida isolate was obtained from the dogs examined. It was found that Candida does not occur in dogs normally and Malassezia was the main colonizing yeast in healthy dogs.
Key words: Candida; dog; incidence; Malassezia; mycoses; skin

\section{INTRODUCTION}

The most frequent fungal infections in animals are dermatophytosis and dermatomycosis. Dermatophytes are keratinophilic and keratinolytic fungi, characterized by a high affinity to keratin-containing tissues. In dogs, dermatophytosis are most often caused by genera of Microsporum and Trichophyton [1]. Dermatomycosis are also very frequently diagnosed in veterinary medicine, especially those caused by yeast of the genera of Malassezia and Candida. The yeasts of these genera are part of an animal's normal flora. However, despite being saprobes, there have been many reports of infections caused by these microorganisms, which present different clinical manifestations.

In dogs, Malassezia spp. have been associated with otitis externa and dermatitis $[27,29,31]$ and as a possible aggravating factor in the physiopathology of corneal ulcers [33]. The predisposing factors that determine the formation of Malassezia mycoses include: the alteration of the host's 
immune system, greasy skin, high temperature, humidity, corticosteroid treatment and others [2]. Currently, the genus Malassezia includes 16 species: M. globosa, M. restricta, M. slooffiae, M. obtusa, M. furfur, M. sympodialis, M. japonica, M. yamatoensis, M. dermatis, M. pachydermatis, M. caprae, M. equina, M. nana, M. cuniculi, M. brasiliensis and $M$. psittaci $[7,8]$.

Several reports have shown that Candida spp. are also important pathogens in dogs, being related to: urinary infections [32], endophthalmitis [26], cutaneous lesions [28], and systemic infections [6]. The group with the most relevant clinical species includes: C. albicans, C. parapsilosis, C. glabrata, C. orthopsilosis, C. metapsilosis, C. tropicalis, C. viswanathii C. tropicalis, C. krusei, C. guilliermondii, C. lusitaniae, C. dubliniensis, C. pelliculosa, C. kefyr, C. lipolytica, C. famata, C. inconspicua, and C. rugosa [13, 37]. The transition to pathogenicity in Candida species, mainly in C. albicans, occurs generally due to the host's immunological condition, leading to a microbiota unbalance, which is associated with important virulence factors, such as biofilm formation and hydrolytic enzymes production, and promotes fungal dissemination to other sites and organs. The clinical manifestations vary from a localized mucosal or skin infection to disseminated disease [23].

The purpose of this study was to compare the occurrence of Malassezia and Candida yeasts on the skin and the mucous membranes in healthy dogs.

\section{MATERIALS AND METHODS}

\section{Animals and samples}

The survey was carried out on 70 healthy dogs in good general health with no history of skin or ear diseases and with no antimicrobial treatments in the past 12 months. The ages of animals ranged from 4 months to 10 years. From each dog, four samples originating from four different anatomical sites of the body (external ear canal, interdigital area, skin of the axilla and of the neck) were collected by a standard swab method. A sterile cotton swab soaked in sterile saline was used to rub against the skin surface, with continuous rotation of the swab over at least a 10 seconds period. In addition, 20 dogs had samples taken also from oral and rectal mucosae.

\section{Cultivation and microscopy}

The samples were inoculated on specific media for the culturing of Malassezia and Candida: Sabouraud dextrose agar with chloramphenicol (SCH) (HiMedia Laboratories Pvt. Ltd., Mumbai, India), Modified Leeming \& Notman agar medium (MLNA) [25], and Modified Candida-Chrom agar (HIT) with Tween 40 [21]. The plates were incubated at $32{ }^{\circ} \mathrm{C}$ for 7 days. All yeast cultures were identified to the genus level by using conventional mycological methods, including the examination of both macroscopic appearance of the colonies (colonies shape, texture and colour) and the microscopic cell morphology (cell size, shape and budding characteristics). In order to assess the lipid dependency, the colonies were subcultured on Sabouraud dextrose agar. Each isolate was stained by Gram and examined by microscopy for the presence of the typical Malassezia and Candida yeast cells. More detailed identification of Malassezia was performed according to Kaneko et al. [20]. Reference strains M. pachydermatis (CBS 1879, Utrecht, Holandsko) (Fig. 1) and C. albicans (CCM 8512, Brno, CR) (Fig. 2) were used as positive controls.

\section{RESULTS}

Forty four out of 70 examined dogs were scored positive for Malassezia spp. (62.9\%). Out of all of the samples only one was identified as a Candida isolate which was obtained from the rectal mucosa and identified as C. albicans. No Candida yeasts were found on the skin. From the samples, a total of 84 Malassezia isolates were obtained. All 84 Malassezia isolates were identified as M. pachydermatis (Fig. 3). The information about the occurrence of yeasts on the skin in the dogs is presented in Table 1 . Seventy three isolates were collected from the skin of the following body sites: 36 isolates - external ear canal (51.4\%); 20 isolates - interdigital area (28.6\%); 10 - axilla (14.3\%); and 7 - neck (10.0\%). Six Malassezia cultures (30.0\%) were obtained from the oral mucosa and five $(25.0 \%)$ from the rectal mucosa (Table 2).

\section{DISCUSSION}

In dogs, yeasts preferably colonize moist areas such as mucous membranes, mucocutaneous junctions, skinfolds, 


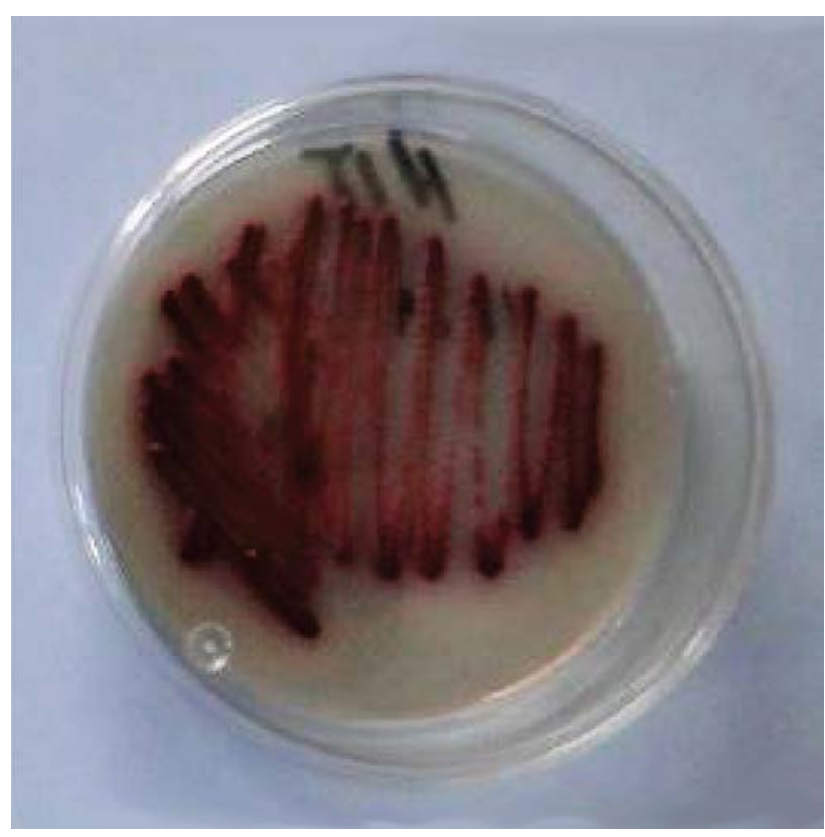

Fig. 1. Cultivation of M. pachydermatis reference strain on HIT

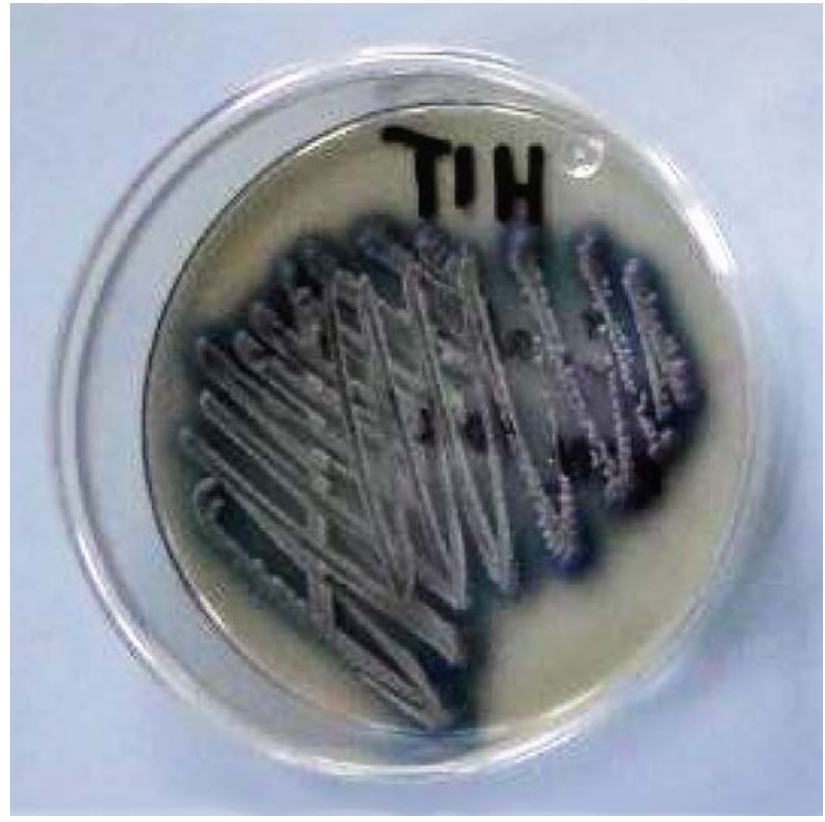

Fig. 2. Cultivation of C. albicans reference strain on HIT

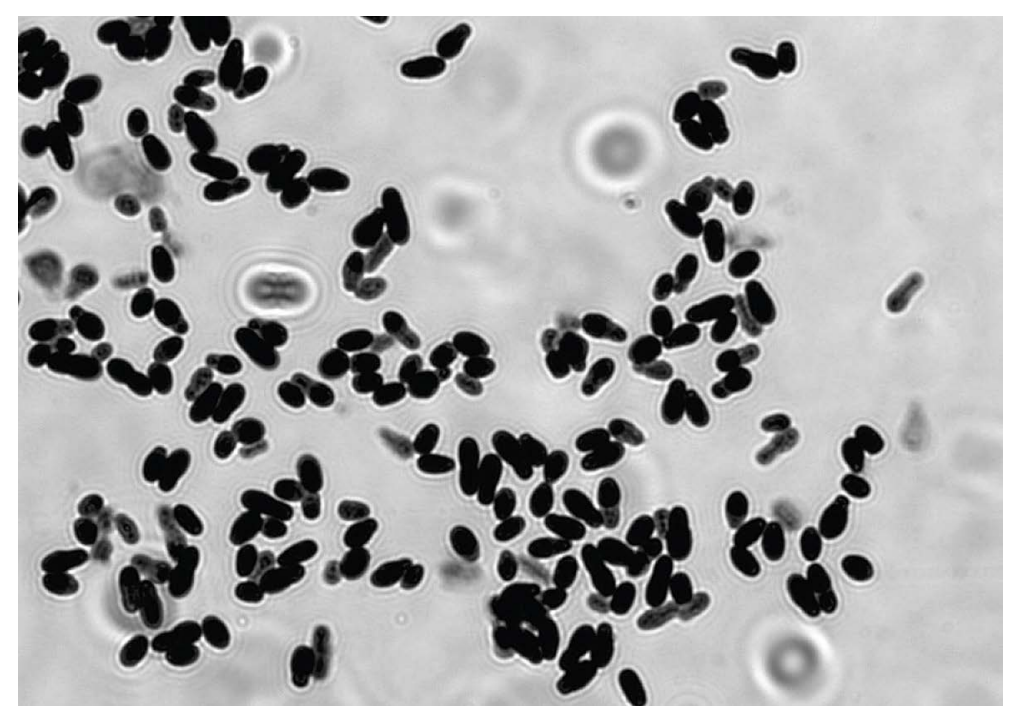

Fig. 3. Cells of M. pachydermatis isolate (Axio Observer Z.1, Magn. $\times 1000$ ).

Table 1. Presence of Malassezia and Candida yeasts on the skin in dogs (70 animals)

\begin{tabular}{lcc}
\hline $\begin{array}{c}\text { Examined } \\
\text { body sites }\end{array}$ & $\begin{array}{c}\text { Number of } \\
\text { Malassezia isolates }\end{array}$ & $\begin{array}{c}\text { Number of } \\
\text { Candida isolates }\end{array}$ \\
\hline External ear canal & $36(51.4 \%)$ & $0(0 \%)$ \\
Interdigital area & $20(28.6 \%)$ & $0(0 \%)$ \\
Skin of the axilla & $10(14.3 \%)$ & $0(0 \%)$ \\
Skin of the neck & $7(10.0 \%)$ & $0(0 \%)$ \\
\hline Total & $\mathbf{7 3}$ & $\mathbf{0}$ \\
\hline
\end{tabular}

Table 2. Presence of Malassezia and Candida yeasts on the mucous membrane in dogs (20 animals)

\begin{tabular}{lcc}
\hline $\begin{array}{c}\text { Examined } \\
\text { body sites }\end{array}$ & $\begin{array}{c}\text { Number of } \\
\text { Malassezia isolates }\end{array}$ & $\begin{array}{c}\text { Number of } \\
\text { Candida isolates }\end{array}$ \\
\hline Oral mucosa & $6(30.0 \%)$ & $0(0 \%)$ \\
Rectal mucosa & $5(25.0 \%)$ & $1(5.0 \%)$ \\
\hline Total & $\mathbf{1 1}$ & $\mathbf{1}$ \\
\hline
\end{tabular}


interdigital areas and ears. Candida and Malassezia species are ubiquitous organisms existing almost exclusively as commensal organisms, which rarely become pathogenic [18]. A range of skin micro-environmental factors, such as the bacterial microbiota present, $\mathrm{pH}$, salts, immune responses, biochemistry, and physiology, may play a role in the adherence and growth of yeasts, favouring distinct genotypes depending on the geographical area and/or the skin sites [17].

Currently, the results of scientific studies on Candida yeasts in animals vary. Brito et al. [5] and Cleff et al. [11] stated, that Candida genus is considered to be part of the microbiota of dogs. We did not identify Candida yeasts on the skin of our examined dogs. This give rise to the question about Candida presence/absence on the skin of dogs. Lee et al. [24] asserted that the presence of Candida in a dog is always the expression of a pathologic state and of its intrinsic pathogenicity. Our results indicate that Candida spp. is not a member of the normal skin flora. We identified one Candida isolate from the rectal mucosa. Candida yeasts colonize mostly mucous membrane [16, 34, 35 ] and their occurrence on the skin is not common and may be bound with outbreak of a disease.

The most Malassezia affected animal is the dog [31]. In general, Malassezia yeasts are associated with the skin and mucous membranes in healthy and diseased dogs $[9,10$, $22,30,31]$. Even $62.9 \%$ of the dogs are positive for Malassezia yeasts. In our study, M. pachydermatis was isolated as the sole species in all dogs examined. Generally, lipophilic M. pachydermatis is the main colonizing yeast in healthy and also in diseased dogs [31]. Only a few times lipid-dependent Malassezia yeasts (M. furfur, M. nana and M. obtusa) were identified $[11,12,14,15,36]$.

In our group of dogs, the external ear canal was the most frequently colonized (51.4\%). However, $\mathrm{Nardoni}$ et al. [30] appointed the frequency of Malassezia isolation: interdigital area $(70.7 \%)$, ears $(63.4 \%)$, axilla $(23.8 \%)$, and perineum and anus (19.0\%). Cafarchia et al. [9] stated that the perianal region is the most frequently colonized and in the ear canal is $12.1 \%$ of all Malassezia. But Ku ma r et al. [22] detected Malassezia yeasts in $39.39 \%$ of healthy ears and Campbell et al. [10] represented $17 \%$ prevalence of Malassezia in healthy ears. The occurrence of Malassezia on the different body sites varies and depends on: the group of examined dogs, season, type of hair and ear and other factors.
Malassezia yeasts have been isolated from different mucosal sites from healthy dogs. In our group, Malassezia occurred on the oral mucosa in $30.0 \%$ of the samples and on the rectal mucosa in $25.0 \%$ of the samples. Haj sig et al. [19] isolated this yeast also from the anus, anal sacs, and vagina of healthy dogs. B ond et al. [4] recovered $M$. pachydermatis from the anus and mouth of $27.5 \%$ of healthy dogs of various breeds. However, Bond and Lloyd [3] suggested that in some cases, mucosal colonization may be secondary to skin proliferation of the lipophilic yeasts.

In conclusion, Candida spp. does not occur on the skin in healthy dog normally. The presence of this yeast is linked rather with the mucosae. On the skin and mucosa is the highest occurrence of Malassezia yeasts and M. pachydermatis remains the most prevalent species in the healthy dog.

\section{ACKNOWLEDGEMENT}

This study was supported by the Slovak Research and Development Agency under the contract No. APVV-15-0377.

\section{REFERENCES}

1. Blaszkowska, J., Wójcik, A., 2012: Current problems concerning parasitology and mycology with regard to diseases of the skin and its appendages. Pol. Parasitol. Soc., 58, 111-123.

2. Bond, R., Ferguso, E.A., Curtis, C.F, Craig, J.M., Lloyd, D. H., 1996: Factors associated with elevated cutaneous Malassezia pachydermatis populations in dogs with pruritic skin disease. JSAP, 37, 103-107.

3. Bond, R., Lloyd, D. H., 1997: Skin and mucosal populations of Malassezia pachydermatis in healthy and seborrheic Basset Hounds. Vet. Dermatol., 8, 101-106.

4. Bond, R., Saijonmaa-Koulumies, L.E. M., Lloyd, D. H., 1995: Population sizes and frequency of Malassezia pachydermatis at skin and mucosal sites on healthy dogs. J. Small Anim. Pract., 35, 147-150.

5. Brito, E.H., Fontenelle, R.O., Brilhante, R.S., Cordeiro, R. A., Monteiro, A. J., Sidrim, J. J., Rocha, M.F., 2009: The anatomical distribution and antimicrobial susceptibility of yeast species isolated from healthy dogs. Vet. J., 182, 320-326.

6. Brown, M. R., Thompson, C. A., Mohamed, F. M., 2005: Systemic candidiasis in an apparently immunocompetent dog. J. Vet. Diagn. Invest., 17, 272-276. 
7. Cabañes, F.J., Coutinho, S.D., Puig, L., Bragulat, M.R., Castellá, G., 2016: New lipid-dependent Malassezia species from parrots. Rev. Iberoam. Micol., 33, 92-99.

8. Cabañes, F.J., Vega, S., Castellá, G., 2011: Malassezia cuniculi sp. nov., a novel yeast species isolated from rabbit skin. Med. Mycol., 49, 40—48.

9. Cafarchia, C., Gallo, S., Romito, D., Capelli, G., Chermette, R., Guillot, J., Otranto, D., 2005: Frequency, body distribution, and population size of Malassezia species in healthy dogs and in dogs with localized cutaneous lesions. See comment in PubMed Commons belowJ. Vet. Diagn. Invest., 17, 316-322.

10. Campbell, J. J., Coyner, K.S., Rankin, S.C., Lewis, T.P., Schick, A.E., Shumaker, A.K., 2010: Evaluation of fungal flora in normal and diseased canine ears. Vet. Dermatol., 21, 619-625.

11. Cleff, M. B., Lima, A.P., Faria, R. O., Meinerz, A. R.M., Antunes, T. A., Araújo, F. et al., 2005: Isolation of Candida spp. from vaginal microbiota of healthy canine females during estrous cycle. Braz. J. Microbiol., 36, 201-204.

12. Crespo, M. J., Abarca, M.L., Cabañes, F. J., 2000: Atypical lipid-dependent Malassezia species isolated from dogs with otitis externa. J. Clin. Microbiol., 38, 2383-2385.

13. Diezmann, S., Cox, C. J., Schönian, G., Vilgalys, R. J., Mitchell, T.G., 2004: Phylogeny and evolution of medical species of Candida and related taxa: a multigenic analysis. J. Clin. Microbiol., 42, 5624-5635.

14. Duarte, E. R., Lachance, M.A., Hamdan, J.S., 2002: Identification of atypical strains of Malassezia spp. from cattle and dog. Can. J. Microbiol., 48, 749-752.

15. Duarte, E. R., Resende, J. C. P., Hamdan, J. S., 2009: Characterization of typical and atypical Malassezia spp. from cattle and dog by random amplified polymorphic DNA analysis. Arg. Inst. Biol., 76, 157-164.

16. Fotos, P. G., Hellstein, J. W., 1992: Candida and candidiasis. Epidemiology, diagnosis and therapeutic management. Dent. Clin. North. Am., 36, 857-878.

17. Gaitanis, G., Magiatis, P., Hantschke, M., Bassukas, I.D., Velegraki, A., 2012: The Malassezia genus in skin and systemic diseases. Clin. Microbiol. Rev., 25, 106-141.

18. Greene, C. E., Chandler, F. W., 2006: Candidiasis, torulopsosis, and rhodotorulosis. In Greene, C. E. (Ed.): Infectious Diseases of the Dog and Cat. 3. Philadelphia, Saunders Elsevier, 627-633.

19. Hajsig, M., Tadic, V., Lukman, P., 1985: Malassezia pachydermatis in dogs: significance of its location. Vet. Arch., 55, 259-266.
20. Kaneko, J., Makimura, K., Abe, I., Shiota, R., Nakamura, Y., Kano, R. et al., 2007: Revised culture-based system for identification of Malassezia species. J. Clin. Microbiol., 45, $3737-3742$.

21. Kaneko, T., Makimura, K., Sugita, T., Yamaguchi, H., 2006: Tween 40-based precipitate production observed on modified chromogenic agar and development of biological identification kit for Malassezia species. Med. Mycol., 44, 227-231.

22. Kumar, A., Singh, K., Sharma, A., 2002: Prevalence of Malassezia pachydermatis and other organisms in healthy and infected dog's ears. Israel J. Vet. Med., 57, 145-148.

23. Lagunes, L., Rello, J., 2016: Invasive candidiasis: from mycobiome to infection, therapy, and prevention. Eur. J. Clin. Microbiol. Infect. Dis., 35, 1221-1226.

24. Lee, H. A., Hong, S., Choe, H., Kim, O., 2011: Folliculitis and alopecia with cutaneous candidiasis in a Beagle dog. Lab. Anim. Res., 27, 63-65.

25. Leeming, J.P., Notman, F.H., 1987: Improved methods for isolation and enumeration of Malassezia furfur from human skin. J. Clin. Microbiol., 25, 2017-2019.

26. Linek, J., 2004: Mycotic endophthalmitis in a dog caused by Candida albicans. Vet. Ophthal., 7, 159-162.

27. Machado, M.L.S., Appelt, C.E., Ferreiro, L., Guillot, J., 2003: Otites e dermatites por Malassezia spp. em cães e gatos. Clínica Veterinária, 44, 27-34.

28. Moretti, A., Posteraro, B., Boncio, L., Mechelli, L., Gasperis, E., Agnetti, F., Raspa, M., 2004: Diffuse cutaneous candidiasis in a dog. Diagnosis by PCR-REA. Rev. Iberoam. Micol., 21, 139-142.

29. Morris, D.O., 1999: Malassezia dermatitis and otitis. Vet. Clin. North. Am. Pract., 29, 1303-1310.

30. Nardoni, S., Dini, M., Taccini, F., Mancianti, F., 2007: Occurrence, distribution and population size of Malassezia pachydermatis on skin and mucosae of atopic dogs. Vet. Microbiol., 122, 172-177.

31. Nardoni, S., Mancianti, F., Corazza, M., Rum, A., 2004: Occurrence of Malassezia species in healthy and dermatologically diseased dogs. Mycopath., 157, 383-388.

32. Ozawa, H., Okabayashi, K., Kano, R., Watari, T., Watanabe, S. Hasegawa, A., 2005: Rapid identification of Candida tropicalis from canine cystitis. Mycopath., 160, 159-162.

33. Prado, M.R., Brito, E.H. S., Giraño, M.D., Monteiro, A. J., Sidrim, J.J.C., Rocha, M.F.G., 2004: Higher incidence of Malassezia pachydermatis in the eyes of dogs with corneal ulcer than in healthy dogs. Vet. Microbiol., 100, 115-120.

34. Radosavljevic, M., Koenig, H., Letscher-Bru, V., Waller, J., 
Maloisel, F., Lioure, B., Herbrecht, R., 1999: Candida catenulata fungemia in a cancer patient. J. Clin. Microbiol., 37, 475-477.

35. Santin, R., Mattei, A. S., Waller, S. B., Madrid, I.M., Cleff, M. B., Xavier, M. O. et al., 2013: Clinical and mycological analysis of dog's oral cavity. Braz. J. Microbiol., 44, 139-143.
36. Sihelská, Z., Váczi, P., Čonková, E., 2016: Species composition of Malassezia yeasts in dogs in Slovakia. Berl. Munch. Tierarztl. Wochenschr., 129, 351-354.

37. Yapar, N., 2014: Epidemiology and risk factors for invasive candidiasis. Ther. Clin. Risk Manag., 10, 95-105.

Received November 22, 2016

Accepted January 18, 2017 